\title{
A Resource Assignment Study on Wireless OFDMA Systems
}

\author{
Alex P. da Silva, Ricardo B. dos Santos*, Francisco Rafael M. Lima*, Francisco Rodrigo P. Cavalcanti and Walter \\ C. Freitas Jr.
}

\begin{abstract}
In this article, we study the time-frequency scheduling for Orthogonal Frequency Division Multiple Access (OFDMA) systems. A number of OFDMA schedulers in the literature divides the scheduling problem into two steps: the resource allocation and the resource assignment. While the resource allocation optimizes various metrics, the resource assignment deals mostly with spectral efficiency and is almost service independent. We compare the some resource assignment algorithms in the literature and some proposed by us in fairness, spectral efficiency and ability to mantain the Quality of Service (QoS) requirements.
\end{abstract}

Keywords-OFDMA, resource allocation, resource assignment and radio resource algorithms.

Resumo-Neste artigo estudamos o escalonamento tempo-frequência para sistemas OFDMA. Um número considerável de escalonadores OFDMA na literatura divide o problema de escalonamento em dois passos: a alocação de recursos e o assinalamento de recursos. Enquanto a alocação de recursos otimiza varias métricas, o assinalamento de recursos lida principalmente com a eficiência espectral e é quase independente do serviço. Nós comparamos alguns algoritmos de alocação de recursos na literatura e alguns propostos quanto a justiça, eficiência espectral e a habilidade de atingir os requerimentos de qualidade de serviço.

Palavras-Chave-OFDMA, alocação de recursos, assinalamento de recursos e algoritmos de recursos de rádio.

\section{INTRODUCTION}

Future radio access networks, such as the Long Term Evolution (LTE) project from the 3rd. Generation Partnership Project (3GPP), are being currently specified and developed by telecommunication standard bodies and by the research community. Among the many new features of these systems, the adoption of OFDMA as multiple access technology is almost an unanimity. OFDMA systems offer a high degree of flexibility to Radio Resource Allocation (RRA) algorithms. Due to the frequency selectivity, it is unlikely to find all subcarriers of a given user in a poor state, what characterizes frequency diversity. Moreover, due to the independence of user channels caused by distinct user positions in a cell, the subcarriers in poor channel states for some users, will be in good channel conditions for other users. This is the multiuser diversity.

\footnotetext{
* These students are supported by a scholarship granted by FUNCAP. This work was supported by a grant from Ericsson of Brazil - research branch under ERBB/UFC.18 technical cooperation contract. The authors are from Wireless Telecommunication Research Group - GTEL / Department of Teleinformatics - DETI / Federal University of Ceará - UFC. Emails: \{brauner,rafaelm,walter,rodrigo\}@gtel.ufc.br
}

In OFDMA systems, time-frequency scheduling is generally formulated as an optimization problem whose optimal solution is not usually found in polynomial time. Most of proposed solutions are suboptimal and based on simple heuristics. Examples of criteria include: sum-rate maximization [1], transmit power minimization subject to QoS constraints [2], max-min rate [3] and proportional fairness between User Equipments (UEs) [4].

Through our research in the RRA literature, we have observed that some works [4], [5] have proposed to split RRA problems into two parts: resource allocation and resource assignment (see more details in section II). This is an interesting approach because RRA problems are usually complex and hard to solve analytically.

In some solutions, like the one in [8], the division in two steps makes possible to optimize different system metrics in each step. While the resource allocation step deals with fairness, QoS requirements and other service specific metrics, the resource assignment step can focus mostly with spectral efficiency. As the resource assignment can be independent of the service, knowledge of an efficient resource assignment algorithm can be valuable.

The remainder of the paper is organized as follows. Section II presents the system modeling. Section III describes the algorithms studied in this work. Finally in section V and VI, the numerical results achieved by simulation and the conclusions are provided, respectively.

\section{System MOdELING}

In this work, we model an OFDMA system in the downlink direction with $J$ UEs sharing $N$ contiguous subcarriers of equal bandwidth. As the division of the bandwidth into multiple orthogonal subcarrier and the insertion of the cyclic-prefix should be sufficient to couple with frequency selective fading and inter-symbol interference, subcarriers are modeled as $N$ flat Rayleigh fading channels correlated in time and frequency.

A Base Station (BS) is assumed to have knowledge of the channel gain $g_{j, n}$ of each UE $j$ in each subcarrier $n$ at the current Transmission Time Interval (TTI). The channel gains are assumed to be constant during one TTI.

The channel gain $g_{j, n}$ is defined as

$$
g_{j, n}=10^{\left(\left(G_{f}+G_{s}-L(d)\right) / 10\right)},
$$

where $L[\mathrm{~dB}]$ is the path loss at distance $d[\mathrm{~km}], G_{s}[\mathrm{~dB}]$ is the shadowing gain and $G_{f}[\mathrm{~dB}]$ is the fast fading gain. 
The Signal-to-Interference plus Noise Ratio (SINR) $\gamma_{j, n}$ of UE $j$ in subcarrier $n$ at the current TTI is defined as

$$
\gamma_{j, n}=\frac{g_{j, n} \cdot p_{n}}{\sigma^{2}} \quad \forall j \in \mathcal{J} \quad \forall n \in \mathcal{N},
$$

where $\mathcal{J}$ is the UE set, $\mathcal{N}$ is the subcarrier set, $p_{n}$ is the transmit power in subcarrier $n$ and $\sigma^{2}$ is the Additive White Gaussian Noise (AWGN) plus interference power. We also define

$$
\bar{\gamma}_{j}=\frac{1}{N} \sum_{n \in \mathcal{N}} \gamma_{j, n} \quad \forall j \in \mathcal{J}
$$

as the average SINR among all subcarriers allocated to UE $j$.

The subcarrier allocation problem can be formulated as

$$
\begin{array}{rl}
\max _{\mathbf{X}} & f(\mathbf{X}) \\
& \text { subject to } \\
& \sum_{j \in \mathcal{J}} x_{j, n} \leqslant 1, \quad \forall n \in \mathcal{N},
\end{array}
$$

where $f(\cdot)$ is an objective function, and $\mathbf{X}$ is the assignment matrix whose elements $x_{j, n}$ assume 1 if subcarrier $n$ is assigned to user $j$, and 0 otherwise.

The instantaneous achieved data rate of UE $j$ in subcarrier $n$ is given by

$$
r_{j, n}=F\left(\gamma_{j, n}\right) \cdot x_{j, n},
$$

where $F(\cdot)$ is the link adaptation mapping function.

As fairnes metric we used

$$
\zeta=\sum_{j \in \mathcal{J}} \log \left(\sum_{n \in \mathcal{N}} r_{j, n}\right)
$$

where a farer allocation takes to a higher $\zeta$ [7].

We assume that the resource allocation algorithm has defined a required rate for each UE for the TTI. The resource assignment algorithm will continue to allocate resources the the UE until this rate is achieved or all resources are assigned.

Since the subcarrier allocation is defined by the scheduler, it is assumed that this information is sent via a separate control channel along with the link adaptation information.

\section{Algorithm Description}

For our proposals, we evaluate five assignment algorithms: Random Algorithm (RND), Rate Maximization (RM), Amplitude Craving Greedy (ACG), Descending SORA Assignment (DSORA) and Ascending SORA Assignment ASORA. For all algorithms, we consider that the allocation part has already been performed, that is, the estimate of subcarriers has been made for all users. Thus, we focus only on the assignment part. A comprehensive description of the assignment schemes is listed in the following.

1) Random Algorithm (RND): This algorithm does not implement any intelligent criterion to assign subcarriers to the users. A subcarrier is chosen randomly and assigned to a user also chosen in a random way. The process is repeated until all subcarriers are assigned or all user requirements are achieved.
2) Rate Maximization Algorithm (RM): Rate maximization (RM) is a classical algorithm for OFDMA system whose objective is the maximization of the overall network rate, restricted to the system total power [5]. In this work, we are interested only in the assignment of subcarriers. According to $\mathrm{RM}$, the user with the maximum rate is found over an assignment matrix, which is composed of the rates experienced by each user in each subcarrier. Thus, the corresponding subcarrier is assigned to it. The subcarrier is removed from the pool of free subcarriers. Additionally, if the user requirement is achieved, the user is taken away from the assignment process. The process continues until the pool of free subcarriers are emptied or no more users need to achieve any requirement.

3) Amplitude Craving Greedy (ACG): The Amplitude Craving Greedy (ACG) was originally proposed in [6]. Here, it is an assignment algorithm based on the normalized gain of each user over all subcarriers. Thus, the user gain experienced in each subcarrier is normalized by the maximum gain of that user. The decision variable is then a normalized assignment matrix composed of the normalized gains of all users. ACG works as follows: the user with the maximum normalized gain is found and the corresponding subcarrier is assigned to it. The subcarrier is removed from the pool of free subcarriers. If the rate requirement is achieved by the user, it is taken away from the assignment process. As described before for the other algorithms, the process continues until all subcarriers are assigned or all users requirements are achieved.

4) Descending SORA Assignment (DSORA): The Descending SORA Assignment algorithm was originally conceived for the assignment part of the SORA algorithm [8]. It is based on a priority list of users ordered according to the actual needed resource of the user. Here, the users who require less resources have the priority. The assignment process works as follows: the subcarrier that provides the highest gain among all ones for the user with the higher priority is chosen. Thus, it is assigned to this user and removed from the pool of free subcarriers. The user is moved to the end of the priority queue. The process continues until there is no more subcarriers to be assigned or all user requirements have been achieved.

5) Ascending SORA Assignment (ASORA): The Ascending SORA Assignment algorithm present the same idea of the DSORA algorithm. It is also based on a priority list of users ordered according to their actual needed resource. However, the users who require more resources have the priority. The assignment process is similar to the DSORA one: the subcarrier that provides the highest gain among all ones for the user with the higher priority is assigned to it and taken away from the pool of free subcarriers. The user is then moved to the end of the priority queue. The process continues until the rate requirement of all users are achieved or when all subcarriers are removed from the pool of free ones.

\section{SCENARIO DESCRIPTION}

In this work, we evaluate the assignment algorithms described in Section III over two scenarios. The first one corresponds to a scenario where the algorithms are evaluated 
for a specific load, in order to compare the results with the optimum solution. It is important to mention that due to temporal complexity in the simulation, this solution was determined only for 4 users and 12 subcarriers. The second scenario includes different loads analysis for all studied algorithms.

Table I presents the main parameters used in the system modeling and in the scenarios.

TABLE I

SYSTEM PARAMETERS

\begin{tabular}{|c|c|}
\hline Parameter & Value \\
\hline \multicolumn{2}{|c|}{$\begin{array}{l}\text { System Model } \\
\end{array}$} \\
\hline Central Operating Frequency & $2 \mathrm{GHz}$ \\
\hline Bandwidth per subcarrier & $15 \mathrm{kHz}$ \\
\hline Path loss & $\begin{array}{c}L[d B] \text { at distance d }[\mathrm{km}] \text { is calculated } \\
\text { by } L=128.1+37.6 \log 10(\mathrm{~d})\end{array}$ \\
\hline Shadowing & $\begin{array}{l}\text { Lognormal with standard } \\
\text { deviation of } 8 d B\end{array}$ \\
\hline Fast fading & Typical Urban (TU) model \\
\hline Noise power per subcarrier & $-123.24 \mathrm{dBm}$ \\
\hline Total base station power & $5 W$ \\
\hline Cell radius & $1 \mathrm{~km}$ \\
\hline Number of Snapshots & 1000 \\
\hline Traffic scenario & full buffer \\
\hline Data rate requirement & $70 \mathrm{kbps}$ \\
\hline \multicolumn{2}{|c|}{$\begin{array}{l}\text { Scenario with Optimum Solution } \\
\end{array}$} \\
\hline Number of subcarriers & 12 \\
\hline Evaluated Loads & 4 users \\
\hline \multicolumn{2}{|c|}{ Scenario with different loads } \\
\hline Number of subcarriers & 24 \\
\hline Evaluated Loads & $4,5,6,7,8,9,10,11$ and 12 users \\
\hline
\end{tabular}

\section{RESUlTS}

The performance evaluation of the assignment algorithms is measured in terms of throughput, user satisfaction and fairness. Firstly, we compare the results of the algorithms with the optimal solution for a specific load. Afterwards, we have tested several user arrival rates in order to give an idea of the algorithm's behavior under increasing loads. The available results present insightful information concerning the assignment schemes.

\section{A. Comparison with Optimal Solution}

In this section, we have evaluated the performance of the assignment algorithms for a specific load with respect to the optimal solution, in order to analyze how much the algorithm performances are close to the optimum one. The optimum solution was determined using the brute-force technique. The results for this specific scenario (load of 4 users) are shown in Table II

TABLE II

\begin{tabular}{c|c|c|c}
\hline \hline Algorithm & Throughput [bits/s] & Satisfaction [\%] & Fairness [bits/s] \\
\hline \hline Optimum & $\mathbf{2 4 1 . 1 7}$ & $\mathbf{9 2 . 7 3}$ & $\mathbf{6 8 7 . 4 7}$ \\
\hline$A C G$ & 205.79 & 89.33 & 651.35 \\
\hline$A S O R A$ & 216.35 & 83.13 & 683.30 \\
\hline$D S O R A$ & 215.29 & 84.55 & 686.50 \\
\hline$R M$ & 225.59 & 89.33 & 682.42 \\
\hline$R N D$ & 205.33 & 78.91 & 662.12 \\
\hline
\end{tabular}

We can observe that the ACG algorithm provides the better performance in terms of user satisfaction when compared to the other assignment ones. However, the throughput of the system and the fairness among users for ACG were low. The ASORA, DSORA and RM algorithms presented the better performance in terms of fairness, close to the optimum solution. In particular, RM presents the same user satisfaction of ACG and the better throughput. RND presents the worst performance since it is not incorporate intelligent assignment criteria.

1) Performance for Different Loads: Here, we present a scenario in which the assignment algorithms are evaluated over different loads (4 to 12 users). Figure 1 shows the system throughput for all algorithms.

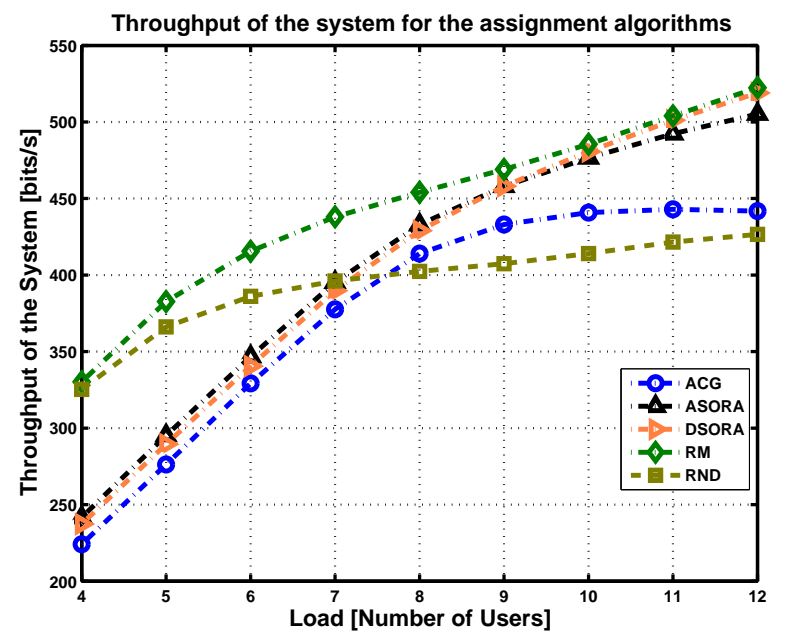

Fig. 1. Throughput of the System.

We can see that the RM algorithm presented the better performance for all loads with respect of the other assignment algorithms, while the ACG algorithm presented low performance for initial loads and a better throughput with regard to the RND algorithm for higher loads, i.e. in its saturation region. ASORA and DSORA presented practically the same performance. As expected, RND present the worst performance for higher loads.

Figure 2 shows the user satisfaction for the algorithms.

ACG presented the better performance followed by the RM algorithm. Obviously, the satisfaction for RND was the lowest since it a random algorithm. For $90 \%$ of satisfied users, we have a capacity of around 7 users for RND, 8 users for ASORA and DSORA, and 9 users for RM and ACG.

We present now the results of fairness in our study, as shown in Figure 3.

From the figure, we can perceive that for RM, DSORA and ASORA the fairness among users was higher than those of ACG and RND. It is important to mention that RND can be considered a fair algorithm in the sense that it does not prioritize specific users.

\section{CONCLUSION}

In this paper we compared different time-frequency schedulers according to fairness, spectral efficiency and user satisfaction. The ACG provided the best user satisfaction but 


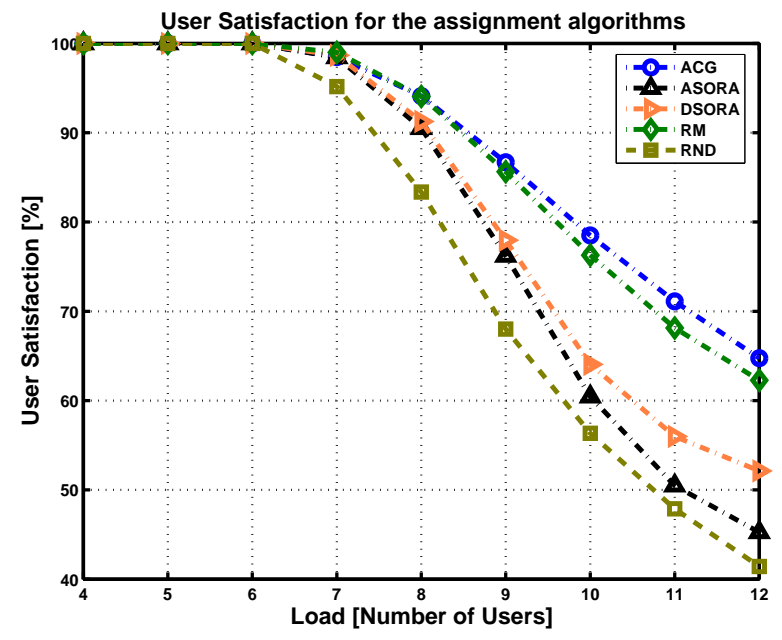

Fig. 2. User Satisfaction.

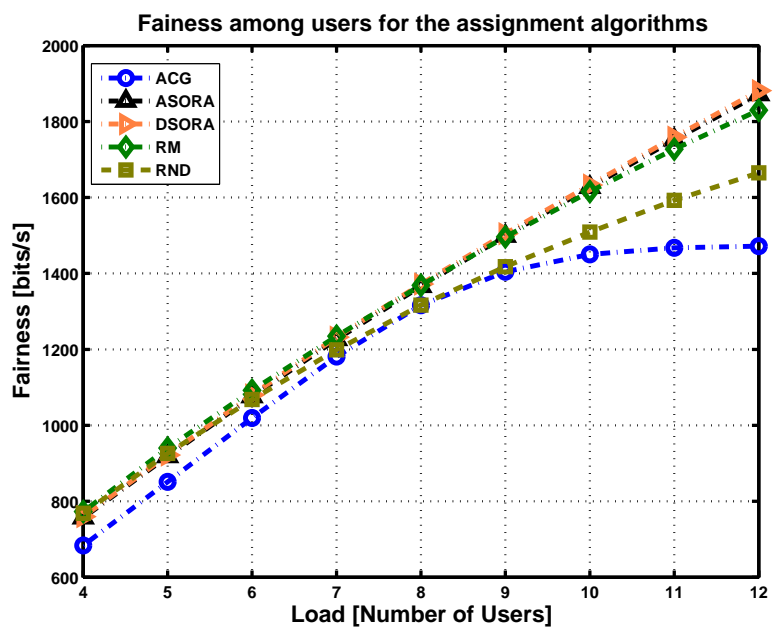

Fig. 3. Fairness among users. provided low spectral efficiency and fairness. The ASORA and DSORA algorithms had good results in fairness and low system throughput and user satisfaction. The RM algorithm, as expected, had the best performance in terms of throughput. The surprise is that, for the studied scenario, RM provided good results in fairness and satisfaction. This behavior is caused by the required rate that limits the number of resources for a UE. This result shows that the RM assignment should have good performance on scenarios where the UEs have little data in the associated buffer.

\section{REFERÊNCIAS}

[1] J. Jang and K. B. Lee, "Transmit Power Adaptation for Multiuser OFDM Systems," IEEE Journal on Selected Areas in Communications vol. 21, no. 2, pp. 171-178, February 2003.

[2] C. Y. Wong, R. S. Cheng, K. B. Letaief, and R. D. Murch, "Multiuser OFDM with Adaptive Subcarrier, Bit, and Power Allocation," IEEE Journal on Selected Areas in Communications vol. 17, no. 10, pp. 1747-1758, October 1999.

[3] W. Rhee and J. Cioffi, "Increase in Capacity of Multiuser OFDM System Using Dynamic Subchannel Allocation," Proc. Vehicular Technology Conference pp. 1085-1089, 2000.

[4] I.C. Wong, Zukang Shen, B.L. Evans and J.G. Andrews, "A Low Complexity Algorithm for Proportional Resource Allocation in OFDMA Systems," IEEE Workshop on Signal Processing Systems, pp. 1-6, Oct. 2004.

[5] J. Gross and M. Bohge, "Dynamic Mechanisms in OFDM Wireless Systems: A Survey on Mathematical and System Engineering Contributions," Telecommunication Networks Group (TKN) Technical Report TKN-06-001, Technical University Berlin, Germany, May 2006.

[6] D. Kivanc and H. Liu, "Subcarrier Allocation and Power Control for OFDMA," Proc. Conference on Signals, Systems and Computers, 2000, vol. 1, pp. 147-151.

[7] F. P. Kelly, A. K. Maulloo and D. K. H. Tan, "Rate Control for Communication Networks: Sahdow Prices, Proportional Fairness and Stability," The Journal of Operational Research Society, vol. 49, nº 03, pp. 237-252, March 1998.

[8] R. B. Santos, F. R. M. Lima, W. C. Freitas Jr., and F. R. P. Cavalcanti, "QoS Based Radio Resource Allocation and Scheduling with Different User Data Requirements for OFDMA Systems," IEEE International Symposium on Personal, Indoor and Mobile Radio Communications vol. $18,2007$. 